\title{
Polarization characteristics of dark- field microscopic polarimetric images of human colon tissue
}

Toru Fujii, Yasuko Yamasaki, Naooki Saito, Masayasu Sawada, Ryo Narita, et al.

Toru Fujii, Yasuko Yamasaki, Naooki Saito, Masayasu Sawada, Ryo Narita, Taku Saito, Heather L. Durko, Photini F. Rice, Gabrielle V. Hutchens, Joceline Dominguez-Cooks, Harrison T. Thurgood, Swati Chandra, Valentine N. Nfonsam, Jennifer K. Barton, "Polarization characteristics of dark-field microscopic polarimetric images of human colon tissue," Proc. SPIE 10890, Label-free Biomedical Imaging and Sensing (LBIS) 2019, 108902J (4 March 2019); doi: $10.1117 / 12.2509000$ 


\title{
Polarization characteristics of dark-field microscopic polarimetric images of human colon tissue
}

\author{
Toru. Fujii*a, Yasuko Yamasaki ${ }^{\mathrm{b}}$, Naooki Saito ${ }^{\mathrm{b}}$, Masayasu Sawada ${ }^{\mathrm{c}}$, Ryo Narita ${ }^{\mathrm{c}}$, Taku Saito ${ }^{\mathrm{c}}$, \\ Heather L. Durko ${ }^{\mathrm{d}}$, Photini F. Rice ${ }^{\mathrm{e}}$, Gabrielle V. Hutchens ${ }^{\mathrm{e}}$, Joceline Dominguez-Cooks ${ }^{\mathrm{e}}$, Harrison \\ T. Thurgood ${ }^{e}$, Swati Chandra ${ }^{e}$, Valentine N. Nfonsam ${ }^{e}$, Jennifer K. Barton ${ }^{e}$ \\ ${ }^{a}$ Nikon Corp., 471, Nagaodai-cho, Sakae-ku, Yokohama, Kanagawa 244-8533 Japan; ${ }^{b}$ Nikon Corp., \\ Shinagwa Intertcity Tower C, 2-15-3, Konan, Minatoku, Tokyo 108-6290 Japan; ${ }^{\circ}$ Nikon System Inc., \\ 6-3, Nishiohi 1-chome, Shinagawa-ku, Tokyo 140-0015 Japan; ' ${ }^{d}$ ikon Research Corp. of America, \\ 12490 N. Rancho Vistoso Blvd., Oro Valley, Arizona, 85755, United States; ${ }^{\mathrm{e}}$ The Univ. of Arizona, \\ 1401 E. University Blvd. Administration Room 601, Tucson, United States
}

\begin{abstract}
Early detection of cancer through medical imaging has a critical impact on patient survival rates. There are many efforts for detecting early cancer in situ using modalities other than traditional medical optical imaging, which contain additional information over conventional micrographs of surface morphology acquired without staining. We analyzed the Mueller matrix components of human colon tissue obtained with an imaging polarimeter microscope at an illumination wavelength of $442 \mathrm{~nm}$ by principal components analysis in order to separate the traditional non-polarized gray image and to investigate the structure of the parameter space of polarization transformation by tissue. We also analyzed Mueller matrix by mapping it to a coherent matrix and performed eigenvalue analysis. The $1^{\text {st }}$ to $4^{\text {th }}$ principal components contain $99 \%$ of the information present in the images; polarization information contributes less than $10 \%$ of the information in the Mueller matrix. In one individual, $80 \%$ of the cancer was detected, without the first components which contains traditional non-polarized gray image for traditional diagnosis. Microscopic fine structures were observed, particularly in the $3^{\text {rd }}$ and $4^{\text {th }}$ principal components' score images. The entropy image of corrugated cancer tissue was smoother than that of the traditional gray image. There were several abnormal regions identified in adjacent regions of cancer, whose residues exceeded the noise level of the instrument used.
\end{abstract}

Keywords: polarization, principal component analysis, human colon cancer, dark-field illumination, Mueller matrix, coherent matrix, eigenvalue analysis, entropy

\section{INTRODUCTION}

Early detection of cancer through medical imaging has a critical impact on patient survival rates. Artificial intelligence (AI) used in the field of cancer diagnosis recently demonstrated that convolutional neural networks (CNN) can detect gastric cancer as accurately as an experienced endoscopic doctor ${ }^{1}$. This performance indicates that AI is becoming a powerful tool for analyzing traditional visible medical images.

There are many efforts for detecting early cancer in situ using modalities other than the traditional images, which contain additional information other than conventional micrographs of surface morphology obtained without staining. Fast and minimally invasive optical techniques such as confocal microscopy ${ }^{2}$ and optical coherence tomography (OCT) ${ }^{3}$ are wellsuited for producing detailed morphological characterizations of small $\left(\mathrm{mm}^{2}\right)$ samples.

Polarization interaction is used in diffuse reflectance spectroscopy ${ }^{4}$ and Mueller polarimetry ${ }^{5}$ imaging because polarimetry is sensitive to micrometer and nanometer structures as predicted by Mie scattering theory ${ }^{6}$. Backman et al. ${ }^{7}$ present an optical-probe technique based on light-scattering spectroscopy that is able to detect precancerous and early cancerous changes in cell-rich epithelia. They utilized the characteristics of early cancer cells, in which the nuclei become enlarged, altering the epithelial-cell architecture. The diameter of non-dysplastic cell nuclei is typically $5-10 \mu \mathrm{m}$, whereas dysplastic nuclei can be as large as $20 \mu \mathrm{m}$ across $^{8}$.

*Toru.Fujii@nikon.com; phone 8145 853-8581; fax 8145 853-8533

Label-free Biomedical Imaging and Sensing (LBIS) 2019, edited by Natan T. Shaked,

Oliver Hayden, Proc. of SPIE Vol. 10890, 108902J · C 2019 SPIE

CCC code: $1605-7422 / 19 / \$ 18 \cdot$ doi: $10.1117 / 12.2509000$

Proc. of SPIE Vol. 10890 108902J-1 
Intensive studies related to Mueller matrix macro imaging of human colon tissue for cancer diagnostics were performed by Mueller matrix decomposition to extract the essential polarimetric effects, such as diattenuation, retardation and depolarization, ${ }^{9,10}$.

Polarization imaging with Monte Carlo simulations of backscattering Mueller matrix macro colon-tissue images were performed by Novikova et al. ${ }^{11}$, who measured spectral Mueller matrix images of ex vivo human colon tissue and have demonstrated that light scattering by small scatterers and light absorption are the key factors for observed polarimetric image contrast.

An imaging polarimeter microscope that operates in a backscattering configuration has been developed for tissue classification and early cancer detection application ${ }^{12,13}$. The difference between the mean measured Mueller matrix values of healthy and cancerous human colon tissue agreed with previously reported results.

W. Wang et al. ${ }^{14}$ measured Mueller matrices of histologically sliced samples and demonstrated the ability to use principal components analysis (PCA) derived from Mueller matrix elements for tissue differentiation.

We analyzed the 16 Mueller matrix images, measured by the imaging polarimeter microscope, by principal component analysis (PCA). The information content in each principal component was examined by comparing their correlation to non-polarized gray images, which includes the tissue morphological information typically used in CNN cancer detection. We also mapped each Mueller matrix to a coherent matrix and analyzed the eigenvalues to investigate the polarization transformations induced by human colon tissues.

\section{PRINCIPAL COMPONENT ANALYSIS}

The imaging polarimeter system consists of an episcopic illumination system capable of dark-field illumination and a polarimetric imaging system for measuring the state of polarization (SOP) of scattered light from the tissue sample positioned at the microscope's specimen stage.

Mueller matrix components of human colon tissues samples acquired from 21 patients were measured with the imaging polarimeter microscope ${ }^{13}$ with a $5 \times$ objective at $442 \mathrm{~nm}(\mathrm{FWHM}<3 \mathrm{~nm})$ illumination. Three types of human colon tissue classifications used in this study: tumor, tumor-adjacent tissues taken $5 \mathrm{~mm}$ from the tumor edge, and normal tissues acquired $50 \mathrm{~mm}$ from the tumor edge.

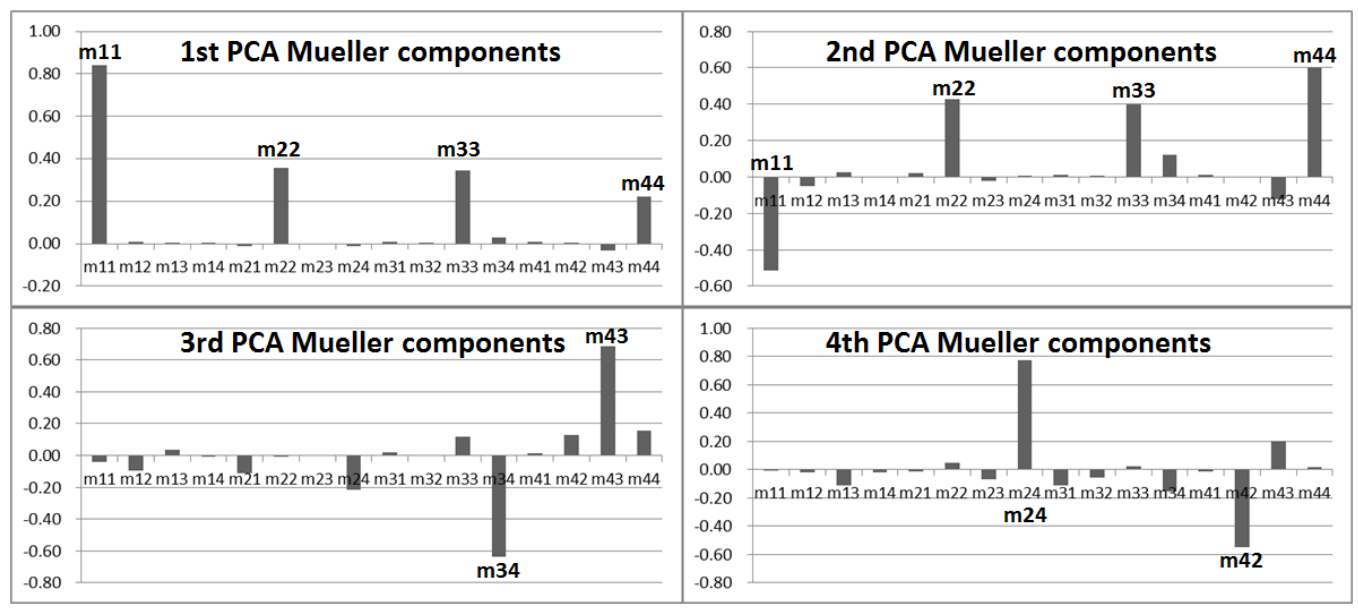

Figure 1. Mueller matrix components of first to fourth principal components of all 21 patients' tissues. The average vector of all images is 0.3 times that of the $1^{\text {st }}$ PCA vector.

Figure 1 shows Mueller components of common PCA axes from the $1^{\text {st }}$ to $4^{\text {th }}$ components analyzed by using 123 Mueller-matrix images acquired from the 21 patients. We also calculate the PCA axes of each of the 123 images and found that $89.3 \pm 6.2 \%$ of Mueller matrix image information were found in the common four-dimensional PCA space.

Figure 2 shows the cumulative contribution rate principal components from $1^{\text {st }}$ to $16^{\text {th }}$ axes. Nearly $99 \%$ of the information is included within the $1^{\text {st }}$ to $4^{\text {th }}$ axis of the PCA space. 
Figure 2. Cumulative contribution rate principal components from $1^{\text {st }}$ to $16^{\text {th }}$. The horizontal axis indicates the number of cumulative components, in which components were added from $1^{\text {st }}$ to $\mathrm{N}$-th components. The vertical axis indicates the value of cumulative components. The $4^{\text {th }}$ cumulative component value is 0.989 .

When we treat four PCA matrices from $1^{\text {st }}$ to $4^{\text {th }}$ as a Jones $\mathrm{N}$-matrix ${ }^{15}$, the $1^{\text {st }}$ to $4^{\text {th }}$ axes correspond to scalar dissipation (extinction) i.e., attenuation (91.2\% information), scalar depolarization (polarization extinction, 4.9\% information), retardation difference between horizontally linearly polarized light and vertically linearly polarized light, i.e., waveplate whose optical axes are vertical and horizontal directions (1.7\%), retardation difference between the two diagonally polarized lights, i.e., waveplate whose optical axes are two diagonal directions $(1.2 \%)$. The $5^{\text {th }}$ and $6^{\text {th }}$ axes (not shown in Figure 1) correspond to transformation from linearly polarized lights to circularly polarized light $(0.4 \%)$, and transformation between vertically and horizontally polarized lights to diagonally polarized lights $(0.3 \%)$.

Wang et al. ${ }^{14}$ reported significant differences in most parameters including retardance, depolarization, linear retardance, linear depolarization and circular depolarization between normal and cancer gastric samples and found that the combination of linear depolarization and linear retardance shows the best overall classification accuracy for gastric samples. In our case for colon tissue, significant differences in polarization parameters correspond to linear depolarization and linear retardance, which are the parameters for gastric cancer classification..

Figure 3 shows four PCA-component images. As discussed above, we can interpret the image as corresponding to gray image, depolarization component image, wave plate component image, and 45-degree rotated wave plate component images respectively. The perturbation of scalar intensity and scalar depolarization are seen in the $1^{\text {st }}$ and $2^{\text {nd }}$ PCA score images, whose modulations are closely related to each other. The trace of intensity remains in $3^{\text {rd }}$ and $4^{\text {th }}$ PCA score images as seen in Patient 5's cancer images, where the image intensity modulation is large.

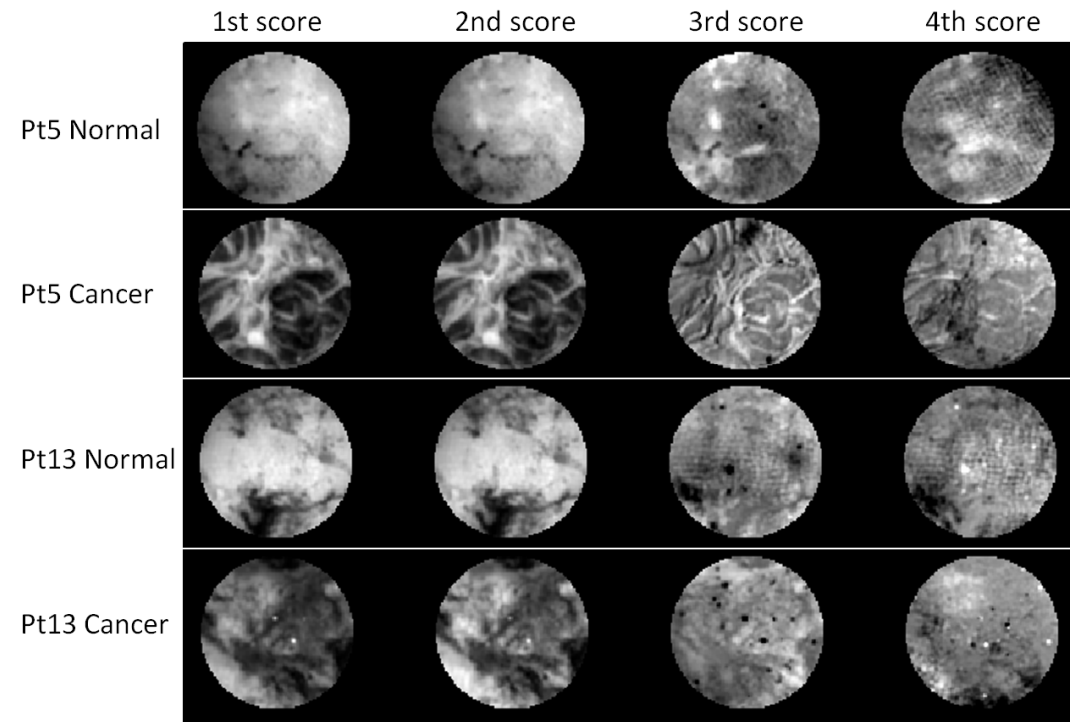

Figure 3. $1^{\text {st }}$ to $4^{\text {th }}$ PCA scores for normal and tumor images of colon tissues removed from Patients 5 and 13 . Field of view is $3.67 \mathrm{~mm}$.

Fine structures observed in the $3^{\text {rd }}$ score and $4^{\text {th }}$ scores have different information from $1^{\text {st }}$ score image, which is assumed to be comparable to a traditional optical grayscale images. This result confirmed the information content in the four- 
dimensional PCA space and the high sensitivity and spatial resolution of the instrument we used for investigating polarization interaction within human colon tissue.

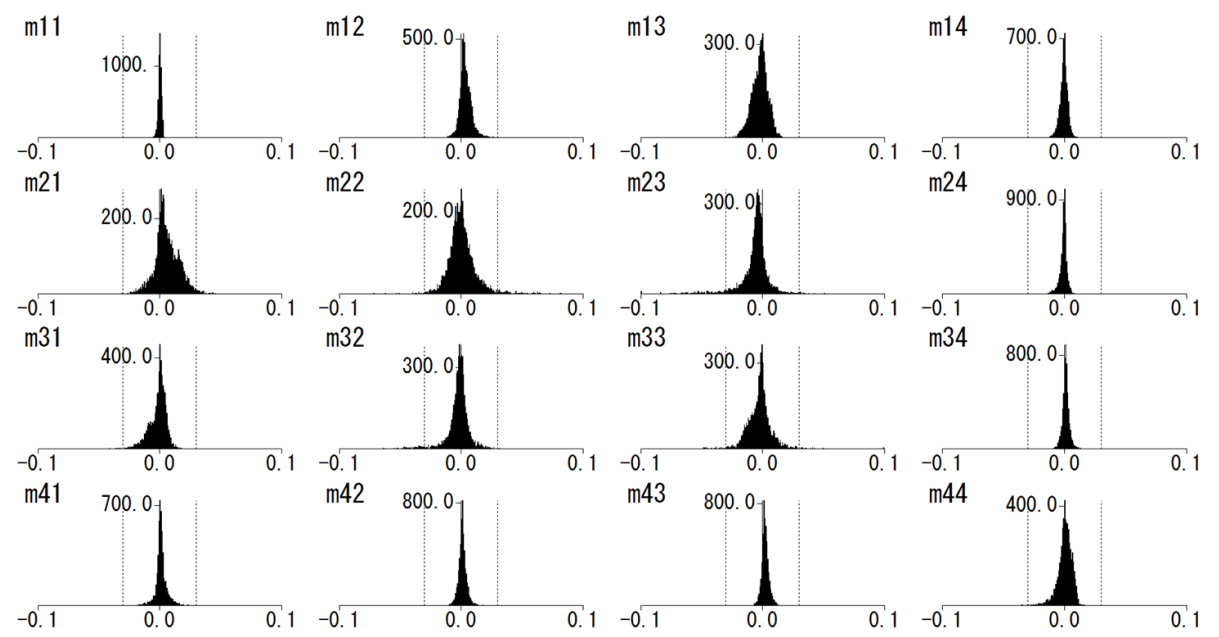

Figure 4. Residue of four-dimensional PCA space Mueller matrix components in histogram form, analyzed from the normal tissue of Patient 2, which is discussed in Section 3. The vertical axis is population of data, i.e., pixel numbers in the image $(\sim 5400$ pixels $)$ and the horizontal axis is the residual value of Mueller matrix components. The two vertical dotted lines are on \pm 0.03 , which corresponds to the noise level $(3 \sigma)$ of the imaging polarimeter microscope.

Figure 4 shows residues typical of the four-dimensional PCA space, whose coordinates are $1^{\text {st }}$ to $4^{\text {th }}$ PCA axes. Vertical dotted lines indicate the $3 \sigma$ noise level of the imaging polarimeter system. These histograms shown in Figure 4 are typical of all three types of tissue. This result also confirmed the low noise level of the instrument we used. We discuss later in Section 4 about a several exceptions where the computed residue is larger than that of typical images.

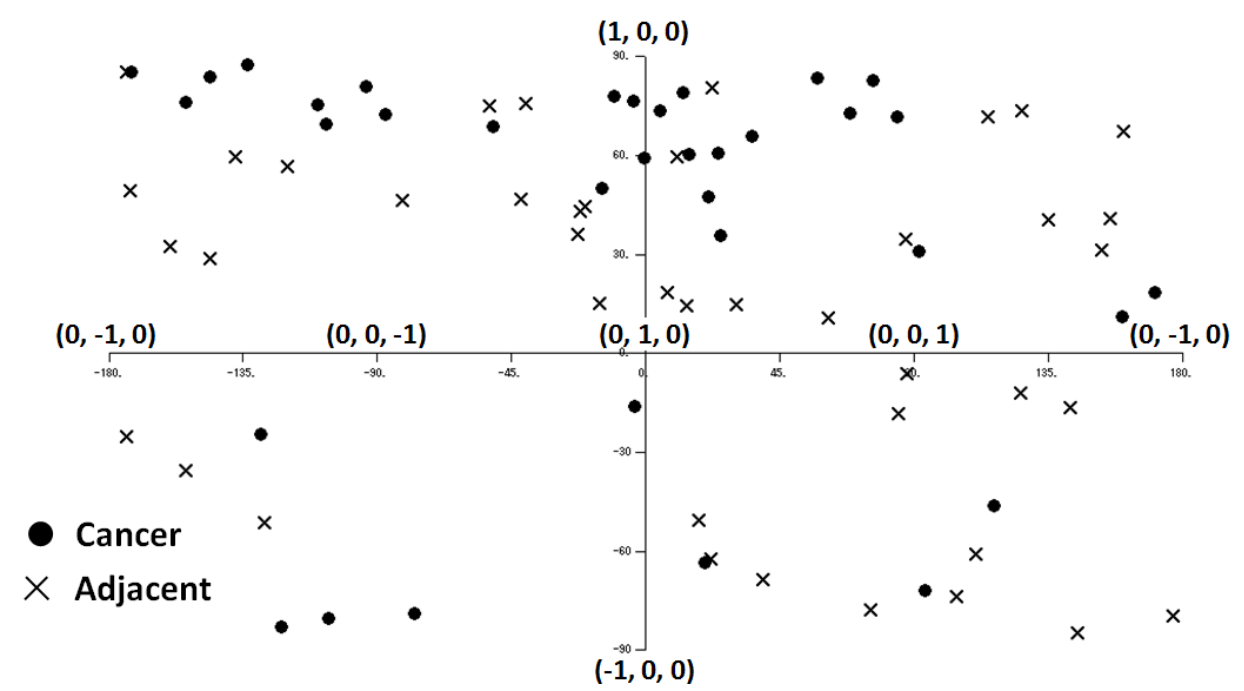

Figure 5. Normalized $2^{\text {nd }}$ to $4^{\text {th }}$ PCA score plots on spherical surface by Mercator projection. The values in parenthesis indicate the position of the normalized value vector $\left(2^{\text {nd }}\right.$ score, $3^{\text {rd }}$ score, $4^{\text {th }}$ score $)$.

Figure 5 shows the scores of $2^{\text {nd }}$ to $4^{\text {th }}$ principal components of tumor from normal, i.e., tumor-normal vector and adjacent from normal of the same individual. The three scores were aligned as a normalized three-dimensional vector as $\left(2^{\text {nd }}, 3^{\text {rd }}, 4^{\text {th }}\right)$. The vectors were then projected on a two-dimensional planer surface by Mercator projection.

Nearly $80 \%$ of the tumor-normal vectors and $65 \%$ of the adjacent-normal vectors of the same individual exist in the northern hemisphere. This result indicates that the $2^{\text {nd }}$ score, which is related to depolarization, mapped with the $3^{\text {rd }}$ and $4^{\text {th }}$ scores is useful for rough tissue classification. 


\section{EIGENVECTOR ANALYSIS AND ENTROPY}

Mueller matrix $m_{i j}$ can be mapped to coherent matrix $C_{i j}$ by (1), where $\eta_{\mathrm{k}}$ is the Dirac matrix ${ }^{5}$.

$$
C_{i j}=m_{i j} \boldsymbol{\eta}_{\mathbf{4}(\mathbf{i}-\mathbf{1})+(j-\mathbf{1})}, 1 \leq \mathrm{i}, \mathrm{j} \leq 4, \boldsymbol{\eta}_{\mathbf{k}}, 0 \leq \mathrm{k} \leq 15
$$

$\mathrm{C}_{\mathrm{ij}}$ contains four eigenvectors, each of which corresponds to four Jones matrices. The target entropy $H_{T}$ is calculated by $(2)^{14,15}$.

$$
\begin{array}{r}
H_{T}=-\sum_{i=1}^{4} P_{i} \log _{4} P_{i} \\
P_{i}=\frac{\alpha_{i}}{\sum_{j=1}^{4} \alpha_{j}} \\
\alpha_{j}: \text { eigenvalue of } \mathbf{C}
\end{array}
$$

Entropy is related to depolarization. There are several methods to quantify entropy, such as the depolarization index ${ }^{16}$, average degree of polarization ${ }^{17}$, and several decomposition-based methods ${ }^{18,}{ }^{19}, 20$. We used entropy because the decomposed Jones matrix with the largest eigenvalue can be used to obtain optical characteristics when the largest eigenvalue is fairly large compared to the remaining eigenvalues.

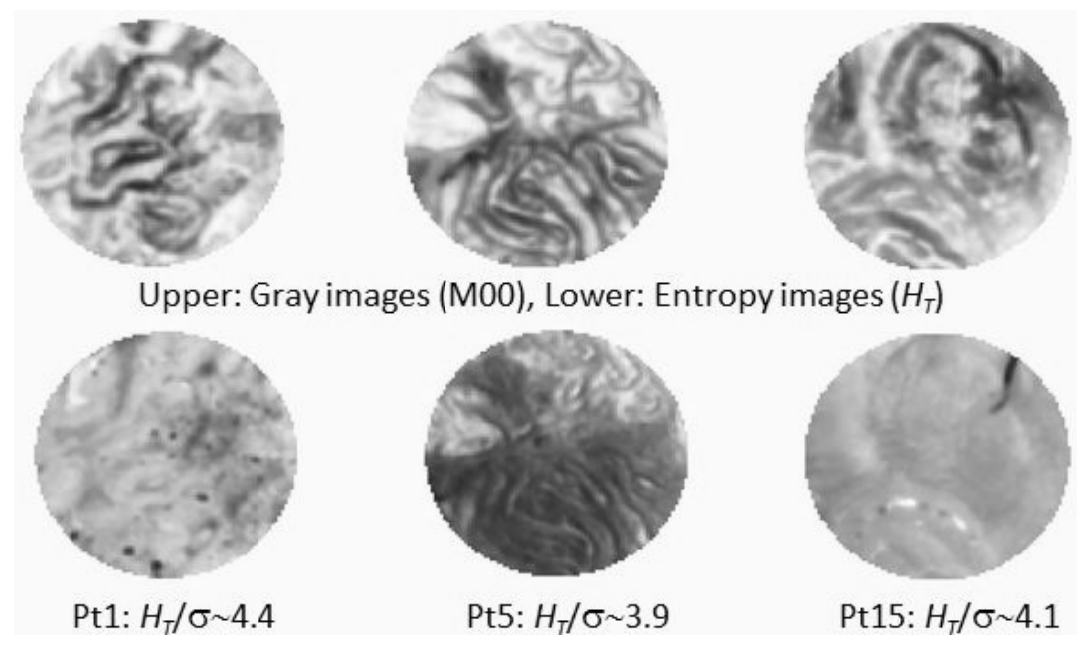

Figure 6. Upper images: Gray scale traditional images of tumor tissue, whose entropy is fairly lower than that of the normal tissue images of the same patient. Lower images: Entropy images of the upper images. A positive index value of entropy/ $\sigma$ indicates the tumor shows lower entropy values than normal tissue as was reported in Ref. 11. The field of view is $3.67 \mathrm{~mm}$.

Figure 6 shows gray intensity traditional images, i.e. $m_{00}$ images, and entropy images of cancer, whose entropy is fairly lower than that of normal tissue adjacent to cancer, which was reported using depolarization previously in the case of early cancer ${ }^{11}$. These gray images are typical adenocarcinoma and can be discriminated easily by eye. There are grayimage-like traces in entropy images, however, the modulation is lower than in the gray images. There is a large trace reduction in the case of Patient 15. The entropy image is far less affected by the surface morphology of human colon tissue than traditional optical images.

The upper three images in Figure 7 show gray images of cancer, whose entropy is not lower. In some cases the entropy is higher than that of normal tissue images of the same patients. Not only entropy cannot be used to discriminate cancer/normal, but the corrugation observed in Figure 3 can be hardly seen in the cancer images. 


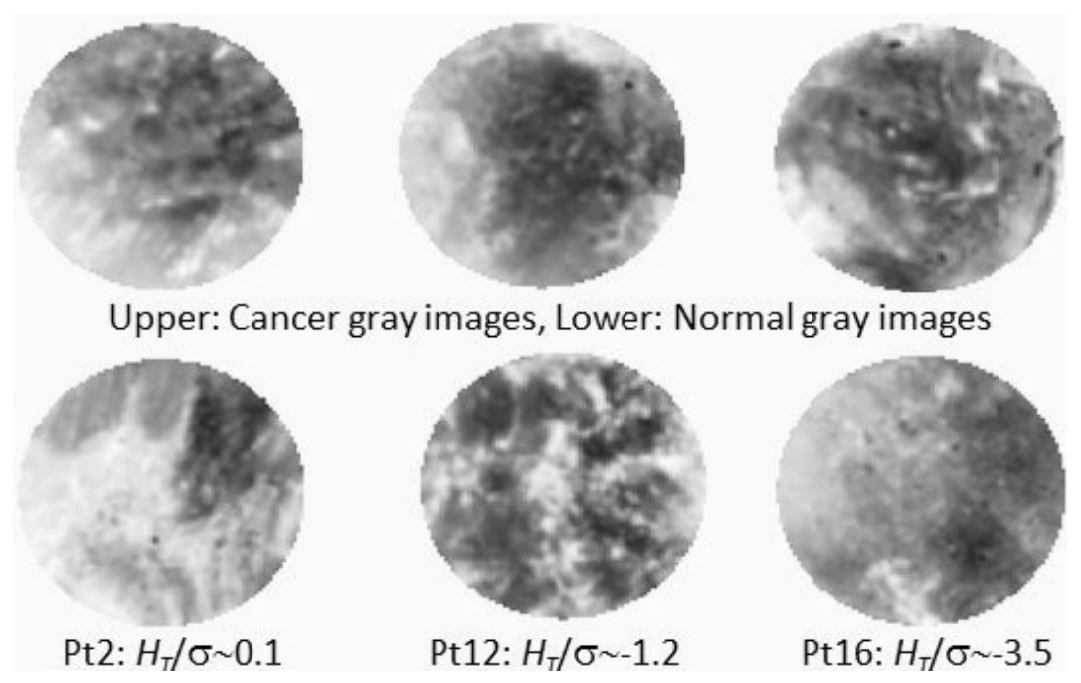

Figure 7. Gray scale images of cancer, whose entropy is not lower (the index above becomes low) than that of the normal images of the same patient. Lower images: Entropy images of the upper images. Field of view is $3.67 \mathrm{~mm}$. Index values are expressed as entropy/ $\sigma$.

Table 1 shows the entropy differences, clinical diagnosis, and pathology as reported by a trained pathologist. Of the 42 totally analyzed images, 29 sites indicated lower entropy than their normal tissue counterparts by the standard deviation from 5400 pixel data in each image. This result is well-correlated with the three-dimensional PCA projected results shown in Figure 3 because the $2^{\text {nd }}$ PCA axis correlates to a degree of depolarization.

Table 1. Response of entropy against each patient with more precise diagnosis. Value of site 1 and 2: difference of mean entropy between tumor image pixels and normal image pixels (5400 pixel each). The unit is the standard deviation acquired from each patient's data. Higher numbers indicate that the tumor entropy is lower than that of normal, which means entropy response is positive and effective. A negative sign means tumor entropy is higher than that of the normal tissue. The clinical diagnosis and pathology results of Patients 17-21 are not available for inclusion in this table.

\begin{tabular}{|c|c|c|c|c|}
\hline Patient No & Site1 & Site2 & Clinical Diagnosis & Pathology from hospital [a] \\
\hline 1 & 4.7 & 4.4 & right colon cecal mass & tubulovillous adenoma \\
\hline 2 & 0.1 & 0.7 & sigmoid colon cancer & municous adenocarcinoma; low grade; $\mathrm{T} 4 \mathrm{aN} 1$ \\
\hline 3 & 1.5 & 1.2 & rectosigmoid colon mass & T4aN1a; focally invasive adenocarcinoma, moderately differentiated \\
\hline 4 & 1.8 & 2.3 & metastatic colon cancer & T3N1a adenocarcinoma \\
\hline 5 & 2.9 & 3.9 & $\begin{array}{l}\text { distal sigmoid colon adenocarcinoma staged as a } \\
\text { T2N0M0 preop }\end{array}$ & $\begin{array}{l}\text { well differentiated invasive adenocarcinoma arising from tubulovillous } \\
\text { adenoma; } \mathrm{pT} 1 \mathrm{~N} 1 \mathrm{~b}\end{array}$ \\
\hline 6 & 5.1 & 2.2 & rectal mass & $\begin{array}{l}\text { colonic mucosa fragments w/ high-grade dysplasia concerning for invasive } \\
\text { disease. }\end{array}$ \\
\hline 7 & 3.6 & 3.0 & rectal adenocarcinoma & ypT4bN0M1c.; G2 ${ }^{*}$ moderately differentiated \\
\hline 8 & 2.9 & -0.2 & Cecal Mass & Tubulovillous adenoma \\
\hline 9 & 0.2 & 0.0 & Rectal cancer with a bowel obstruction & T3N2, invasive poorly differentiated adenocarcinoma \\
\hline 10 & 0.5 & 2.3 & Distal rectal polyp & Tubular adenoma \\
\hline 11 & 2.3 & 4.3 & Distal sigmoid colon cancer & $\begin{array}{l}\text { pT3N0M0, Invasive adenocarcinoma, moderately to poorly differentiated, } \\
\text { involving pericolonic adipose tissue }\end{array}$ \\
\hline 12 & -1.2 & -0.9 & Ascending colon cancer & Sessile serrated adenoma with cytologic dysplasia \\
\hline 13 & 2.4 & 2.9 & Hepatic flexure adenocarcinoma & \\
\hline 14 & -1.9 & -2.4 & $\begin{array}{l}\text { Rectal Cancer, patient } 62 \text { new mass, after } \\
\text { chemoradiation }\end{array}$ & Invasive adenocarcinoma, T4b N1c M1c \\
\hline 15 & 4.2 & 4.1 & Sigmoid colon cancer & Medullary carcinoma, $\mathrm{T} 4 \mathrm{a} \mathrm{N} 2 \mathrm{~b}$ \\
\hline 16 & -2.5 & -3.5 & Rectal cancer post chemotherapy & Moderately differentiated invasive adenocarcinoma, $\mathrm{T} 3, \mathrm{~N} 0$ \\
\hline
\end{tabular}

The largest discrepancy is seen in the patients who received chemoradiation and chemotherapy. This table also indicates that the current clinical diagnosis is not simply related to entropy as calculated from the Mueller matrices. 


\section{RESIDUE OF FOUR PCA COMPONENTS}

The typical histogram of the three types of tissue showed that the residue is inside the dotted line of $\pm 0.03(3 \sigma)$, which is a noise level of the imaging polarimeter microscope. However, we found a few exceptions where the residue is larger than that of typical images, as was discussed in Section 2.
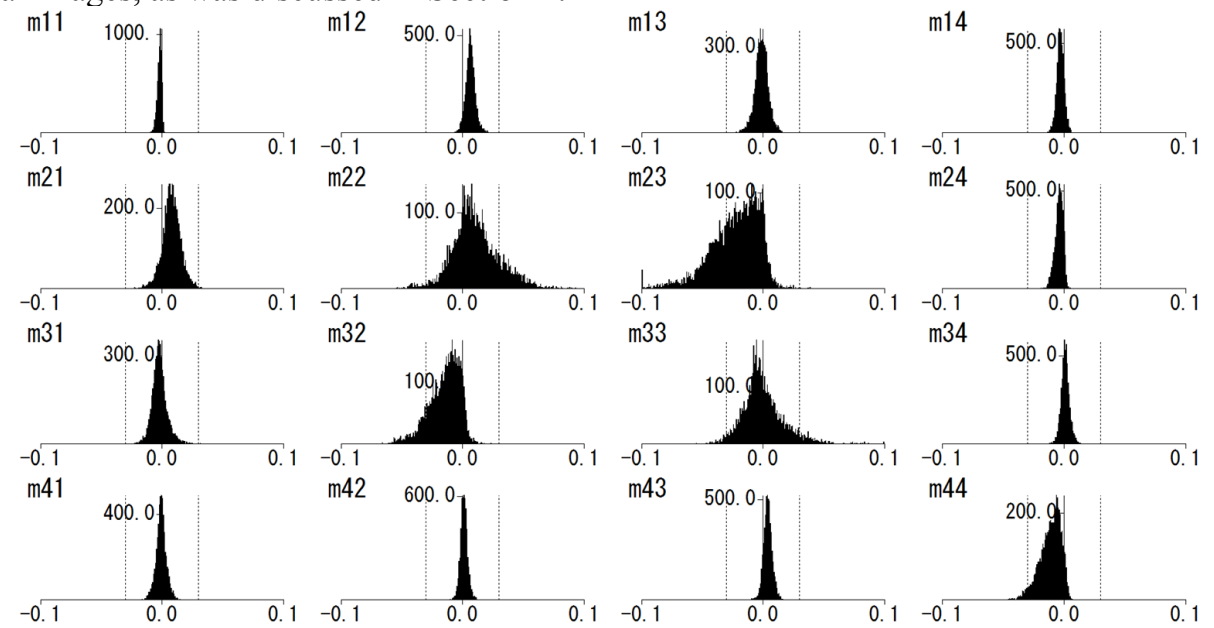

$\mathrm{m} 42$
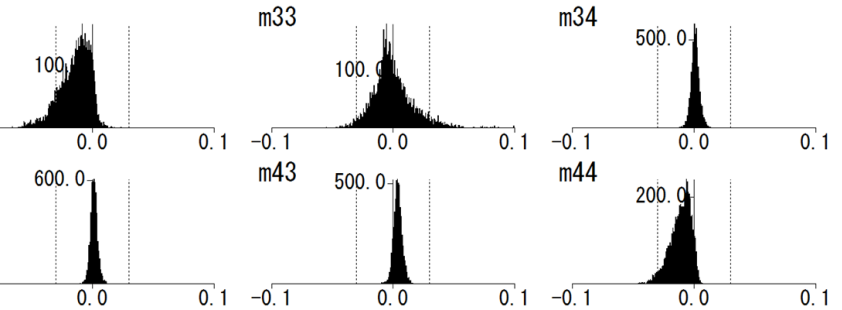

Figure 8. Exception example of residue of Mueller matrix components 4 PCA components analyzed using adjacent image of patient 2. Vertical axis is population of date, i.e., pixel numbers in the image ( $\sim 5400$ pixels) and horizontal axis is value of Mueller matrix components. Two vertical red lines are on \pm 0.03 , which is the noise level $(3 \sigma)$ of the imaging polarimeter microscope.

Figure 8 shows an example of the instances where the residue is larger than the vertical dotted lines. These excerptions were seen in mainly adjacent-tissue images and in one normal-tissue image of Patient 2 . The extra residues could be seen mainly in the Mueller components $m_{22}, m_{23}, m_{32}$, and $m_{33}$. Further experiments, analysis, and simulation of the tumor adjacent tissue must be needed to interpret this phenomenon using an instrument with sufficient spatial resolution.

\section{CONCLUSION}

We measured Mueller matrix images of human colon tissue with an imaging polarimeter microscope at $442 \mathrm{~nm}$, and analyzed the 16 matrix images components by PCA in order to separate the traditional non-polarized gray image information from the Mueller matrix information and to investigate polarization interaction between human colon and illuminated light. Each principal component was compared to non-polarized grayscale images containing the traditional morphological information used in CNN cancer detection. We also analyzed the Mueller matrix by mapping it to a coherent matrix and performed eigenvalue analysis. Up to $99 \%$ of the information in human colon tissue is contained within the first four principal components. Polarization information is contained within the second and subsequent principal components; this information is less than 10 percent of the total information present in the Mueller matrix images. Optical interactions induced by each principal component from first to fourth correspond to scalar dissipation directly related to conventional image, scalar depolarization, and linear phase retardance, respectively. The relative contributions of these components to the total information in the image are $91.2 \%, 4.9 \%, 1.7 \%$, and $1.2 \%$, respectively. Microscopic fine structures observed in the third and fourth scores have different information than what is obtained from traditional optical gray images. The amount of the residue of the four components, whose amount is $1.1 \%$, agrees well with the noise level of $\pm 0.03(3 \sigma)$ in the Mueller components of the imaging polarimeter microscope we used. Nearly $80 \%$ of cancer images were discriminated in the three-dimensional spherical surface formed by the second to fourth principal components, in which non-polarization information is small. There were several abnormal examples in canceradjacent regions where the residues exceeded the noise level of the instrument used. Further investigations into the data acquired from cancer-adjacent tissue will be performed. 


\section{REFERENCES}

[1] Sakai, Y., Takemoto, S., Hori, K., Nishimura, M., Ikematsu, H., Yano, T., and Yokota, H., "Automatic detection of early gastric cancer in endoscopic images using a transferring convolutional neural network", Conf. Proc IEEE Eng. Med Bio Soc., Jul; 2018:4138-4141, (2018).

[2] Pawley, James (Ed.) [Handbook of biological confocal microscopy], Springer US (2006).

[3] Fujimoto, J., G., Pitris, C., Boppart, S. A. and Brezinski, M., E., "Optical Coherence Tomography: An Emerging Technology for Biomedical Imaging and Optical Biopsy", Neoplasia. Jan; 2(1-2): 9-25 (2000).

[4] Zonios, Z., Perelman, L., T., Backman, V., Manoharan, R., Fitzmaurice, M., Dam, J., V. and Feld, M. S., "Diffuse reflectance spectroscopy of human adenomatous colon polyps in vivo", Appl. Opt. 38 (31) 6628-6637, (1999).

[5] Goldstein. D., H., [Polarized Light], CRC Press (2003).

[6] Tuchin, V. V., Wang, L., and Zimnyakov, D. A., [Optical Polarization in Biomedical Applications], Springer Science \& Business Media (2006).

[7] Backman, V., Gurjar, R., Badizadegan, K., Itzkan, L., Dasari, R. R., Perelman, L. T. and Feld, M. S., "Polarized light scattering spectroscopy for quantitative measurement of epithelial cellular structures in situ", IEEE J. Sel. Top. Quantum Electron. 5, 1019 (1999).

[8] Backman, V., Wallace, M., B., Perelman, L., T., Arendt, J., T., Gurjar, R., Mueller, M., G., Zhang, Q., Zonios, G., Kline, E., McGillican, T., Shapsshay, S., Valdes., T., Badizadegani, K., Crawford, J. M., Fitzmaurice, M., Kabani, S., Levin, H. S., Seiler, M., Dasari, R. R., Itzkan, I., Dam, J., V., and Feld, M. S., "Detection of preinvasive cancer cells", nature 406, 35 (2000).

[9] Alali, S. and Vitkin, A., "Polarized light imaging in biomedicine: emerging Mueller matrix methodologies for bulk tissue assessment", J. Biomed. Opt. 20(6), 061104 (2015).

[10] Tuchin, V., V., "Polarized light interaction with tissues", J. Biomed. Opt. 21(7), 071114 (2016).

[11] Novikova, T., Pierangelo, A., Manhas, S., Benali, A., Validire, P., Gayet, B. and De Martino, A., "The origins of polarimetric image contrast between healthy and cancerous human colon tissue", Appl. Phys. Lett. 102, 241103 (2013).

[12] Chen, Z., Yao, Y., Zhu, Y. and Ma, H., "A collinear backscattering Mueller matrix microscope for reflection Muller matrix imaging", Proc. of SPIE 10489 104890M-1 (2018).

[13] Saito, N., Sato, K., Fujii, T., Durko, H., L., Goldstein, G., L., Phillips, A., H., Dominguez-Cooks, J., Hutchens, G., V., Thurgood, H., T., Rice, P., F., Barton, J., K., "Multispectral Mueller matrix imaging dark-field microscope for biological sample observation", Proc. SPIE 10890-46 (2019).

[14] Wang, W., Lim, L. G., Srivastava, S., So, J. B.-Y., Shabbir, A. and Liu, Q., "Investigation on the potential of Mueller matrix imaging for digital staining", J. Biophotonics 9 (4), 364 (2016).

[15] Jones, R., C., "A new calculus for the treatment of optical systems. VII. Properties of the N-matrices," J. Opt. Soc. Am. 38, 671-685 (1948).

[16] Claude, S., R., and Pottier, E., "An Entropy Based Classification Scheme for Land Applications of Polarimetric SAR", IEEE Transactions on Geoscience and Remote Sensing. 35, 68-78 (1997).

[17] Cloude, S. R., "Polarimetry: The characterization of polarization effects in EM scattering", Dr. Thesis University of Birmingham, (1987).

[18] Gil, J., J., and Bernabeu, E., "Depolarization and polarization indices of an optical system" OPTICA ACTA 33, 185-189 (1986).

[19] Chipman, R., A., "Depolarization index and the average degree of polarization", Appl. Opt. 44(13), 2490 (2005).

[20] Lu, S-Y., and Chipman, R., A., "Interpretation of Mueller matrices based on polar decomposition", JOSAA 13(5) 1106, (1996).

[21] Ortega-Quijano, N. and Arce-Diego, J., L., "Mueller matrix differential decomposition", Opt. Lett. 36(10) 1942 (2011).

[22] Ossikovski, R., “Analysis of depolarizing Mueller matrices through a symmetric decomposition”, JOSAA 26(5) 1109 (2009). 